\title{
Evaluation of on-line schedules by distributed simulation*
}

\author{
S. R. Jernigan, S. Ramaswamy, K. S. Barber \\ The Laboratory for Intelligent Processes and Systems, The Department \\ of Electrical and Computer Engineering, The University of Texas at \\ Austin, Austin TX 78712-1084, USA
}

\begin{abstract}
A new algorithm for the distributed simulation and evaluation of on-line schedules is presented. Generally, on-line scheduling has often been restricted to scheduling activities on a single machine or workcell. The exploratory research reported in this paper expands on-line scheduling to encompass several machines or workcells. Branch and bound search techniques are used in the simulation to reduce the number of simulations simultaneously in execution. The algorithm is applied for the distributed simulation of on-line schedules for a manufacturing example.
\end{abstract}

\section{INTRODUCTION}

Several obstacles prevent current assembly and manufacturing lines from capitalizing on the promises of complete automation. Among these are the difficulties in scheduling ${ }^{1}$ resources and ordering processes such that optimal use is made of the current configuration. Fox and Kempf [FoKe85] distinguish the differences between planning and scheduling and suggest that a distinction is both essential and beneficial. While planning is often done off-line, scheduling can be best performed at run-time [FoKe85, XiBe88, ChA186, MeSa91, Lyon90]. Planning is an activity that produces a minimally constrained plan $^{2}$ and provides estimates for raw materials requirements and product yields. Scheduling is an activity that uses the plan developed by the off-line planner and develops a schedule at run-time, incorporating the current state of the factory floor. Such a schedule will be maximally constrained and it will specify the necessary details for executing a schedule. Furthermore, the scheduler can reactivly adapt the schedule in response to unexpected outcomes or states. Previously, on-line scheduling has been restricted to a single machine or workcell. The exploratory research reported in this paper expands on-line scheduling to encompass several machines or workcells. Various representation schemes have been used to represent operational orderings of a plan. These include: (i) AND/OR graphs [FoKe85], [MeSa91], (ii) Hierarchical hypergraphs [XiBe88], (iii) process algebra [Lyon90],

\footnotetext{
* This research was supported in part by the Texas Higher Education Coordinating Board under grant ATP115 .

1 Scheduling can be classified as either on-line or off-line scheduling. In this paper, scheduling is used to refer to on-line scheduling, unless otherwise explicitly stated. For more discussions on scheduling, the reader is referred to [FoKe85] and references therein.

2 A minimally constrained plan is a plan that will not require replanning in the future. Constraints known apriori are used to generate a minimally constrained plan off-line. A maximally constrained plan is a plan that is generated taking into consideration all available constraints. A maximally constrained plan is generated at run-time and can be directly executed.
} 
(iv) directed acyclic graphs (DAGs), (v) Petri Nets [KrSr887], and, (vi) frames [Haye93]. For the example in this paper, a listing of partial orders, a termination set of processes, and a set of previously executed processes will form the part representation. A distributed simulation scheme is used to evaluate possible schedules. The reader is refered to [Jern95] for a detailed survey of the literature on search techniques and simulation.

Finding efficient heuristics to reduce the size of the search space to make the simulation tractable is a primary objective of this work. Although the search is bounded, the number of simulations is large for even moderately complex environments. The significant characteristics of the simulation described in this paper include: (i) Each resource on the factory floor is assumed to have some computational element that can make process decisions with respect to its current status. Typically, this computational element is combined with other such elements in a central process. However, this tight coupling between the central process and the individual machines makes expansion, changes, and upgrades difficult. In the distributed simulation environment described in this paper, the computational element for a machine, the machine's controller, and the physical resource are modeled as a single agent. Given this model, each machine has the added capability to reason by itself. Once the system is decoupled in this manner, machines may now reason very differently. For instance, one machine may use intensive geometric reasoning while another may use simple semantic reasoning. The part and schedule representations are the only constraints on the method of reasoning for each agent, (ii) Upgrades can be incorporated into the scheduling unit of a single machine without affecting the rest of the system. Thus, each module must have a minimum set of constraints to be integrated with the system, while allowing the maximum amount of freedom to perform its job in the manner it chooses. On the other hand, the modularity introduces a serious architectural problem. Since no machine can assume anything about the operation of the other machines, each module cannot directly assess the extended results of its actions. In essence, each machine is blind with respect to other machines or its role in the system. The absence of a global view makes the application of heuristics considerably more difficult, (iii) The maintanence of a central time keeping process helps to provide a global view of the simulation, (iv) The simualtion considers the generation of on-line schedules for a group of machines or workcells, and, (v) The method proposed has the flexibility to exploit process dependent and process independant heuristics [Jern95].

This paper is organized as follows. Section 2 presents the new distributed environment. Section 3 analyzes a complete trace of a simulation. Section 4 concludes the paper with issues for future development and research.

\section{THE NEW SIMULATION METHOD}

In this research, simulation is employed to both generate and evaluate schedules. Simulated schedules are generated and evaluated based on the processes performed, in comparison to the input plan, and the current state of the system. The number of potentially feasible schedules to simulate is a dynamic function of the changing system state. The state of the system cannot be predicted off-line due to unexpected and random events. An on-line simulation of schedules can react to the current state when evaluating plans. The primary modification made to basic sequential simulators [Misr86] for this research is the ability to handle points of nondeterminism. In each new simulation created, one of the valid responses to the event that triggered the duplication will be simulated. Each of these simulations can continue in parallel since they are completely duplicated.

\subsection{The Central Algorithm}

Each simulation can be in one of four states: ready (to process an event), waiting (for the previous event to finish processing), terminated, successfully completed. While the simulation 
is active, it cycles between the ready and the waiting states. When a simulation reaches a point of non-determinism, all the possible choices must be considered. At this point, a message is sent to the central process that requests the simulation to be split into a number of duplicate simulations. The central processor duplicates the event list for the simulation and signals all agents to duplicate any information held for that simulation. The requesting agent can then reflect each possible path in one of the duplicate simulations. The algorithm for the central process is given in Figure 1.

$\mathrm{T}=\mathrm{T} 0, \mathrm{~T} 1, \ldots \mathrm{T}(\mathrm{x}-1)$

where $\mathrm{x}$ is the number of simulations and Tw is the global clock of simulation $w$. The global clock can be defined as: $\mathrm{Tw}=\mathrm{MIN}(\mathrm{t})$

where $\mathrm{t}=\mathrm{t} 0 \mathrm{t} 1, . . \mathrm{t}(\mathrm{y}-1)$, $\mathrm{y}$ is the number of factory floor processes, and ts is the next expected time in which factory floor process $s$ will generate an event.

Central

Create simulation $0, T=0$, invalidate all expected event times

Send A Start Simulation Message

Bound $=\infty$

Repeat

From subset of simulation that have all agents registered, $\mathrm{T}^{\prime}$,

find the simulation with $\mathrm{Tv}=\mathrm{MNN}\left(\mathrm{T}^{\prime}\right)$

Advance $\mathrm{Tv}$ to $\mathrm{MIN}(\mathrm{t})$

If $\mathrm{Tv}>$ Bound then

Locally delete simulation $\mathrm{v}$

Broadcast delete simulation v message

Notify agent with registered time Tv of the current time and mark that agent as invalid

If any validation messages have been received for agent $\mathrm{z}$ in simulation $\mathrm{q}$

For simulation $\mathrm{q}$

register new time for $\mathrm{Tz}$

invalidate any other agents included in the message

mark agent $\mathrm{z}$ as valid

If any delete messages have been received for simulation $b$

Locally delete simulation $b$

Broadcast delete simulation $b$ message

If any split message received

Create the simulation to the correct number of new simulations

Broadcast split message with old and new simulations

Search any simulations that have all registered times as $\infty$ and no work in progress

If $T h$ is $<$ Bound then Bound $=$ Th and save simulation $h$

Until no simulations are still running.

Figure 1 The Central Algorithm.

\subsection{Floor Agents}

Until this point in discussion, it was assumed that all factory floor processes are identical. In actuality, there exist four distinct groups of factory floor process. Each represents a group of physical entities on a real factory floor. These are: (i) queues, (ii) inspection stations, (iii) materials handling processes, and, (iv) machines.

- Queues: Queues are the simplest form of floor process and represent deterministic material handling methods. For instance, a conveyor belt can be modeled deterministically because it cannot change the state or ordering of the parts it contains. Queues must be specified with upstream and downstream neighbors as well as capacity and transfer rates. 
- Inspection stations: The simulator assumes that no errors will be generated by the inspection station. The inspection stations may introduce delays and exhibit some buffering. Inspection stations can be a form of subgoaling to reduce the search space.

- Materials handling process: Material handling processes are non-deterministic. For instance, a robotic manipulator may have a choice of one of many machines to which it can deliver the part. The material handling process must know its downstream and upstream neighbors. Furthermore, its capacities and capabilities must be thoroughly defined.

- Machines: Machines are floor agents that can cause a change in the state of the product and are the most complicated floor process. Machines must match their own capabilities with the needs of the current part. This involves solving the partial ordering problem. The specific algorithm used depends on the part representation. Each machine must also know its capabilities as well as its upstream neighbor, downstream neighbor, and central process.

\section{AN EXAMPLE}

\subsection{System Description}

This section provides a complete trace of a single simulation for a sample computer assembly factory. In the example, three separate workstations are being assembled. The workstations produced by the factory can have several different configurations. New worsktations start at the beginning of the factory as an empty case. Each workstation must have a motherboard (MB) and a power supply (PS). The motherboard must be installed first. Cards that are available for installation are: a network interface card (NI), a monochrome monitor card (BW), a color monitor card (CR), and a drive controller (DC). Any subset of these cards may be installed in a workstation. Three drives are available for installation: a hard drive (HD), a floppy drive (FD), and a tape drive (TD). Drives have to be installed after the installation of the drive controller.

Workstation $\mathrm{A}$ is a diskless workstation with a motherboard, power supply, monochrome monitor card, and a network interface card. Workstation B is a standalone workstation with a motherboard, power supply, drive controller, monochrome monitor card, hard drive, and floppy drive. Workstation $\mathrm{C}$ is a high-end workstation with a motherboard, power supply, drive controller, color monitor card, network interface card, hard drive, and tape drive. Table 1 summarizes the configuration of each workstation. At the given moment, $t=0$, workstation $\mathrm{A}$ has just arrived and the other two workstation's assembly are already in progress. Workstation $\mathrm{B}$ is having a motherboard installed and has 2.4 time units remaining on the operation. Workstation $\mathrm{C}$ already has a motherboard and power supply and has 3 time units remaining on the installation of its color monitor board.

Table 1 Workstation configurations.

\begin{tabular}{l|l|l|l|l|l|l|l|l|l|} 
Workstation & MB & PS & NI & BW & CR & DC & HD & FD & TD \\
\hline A & $\sqrt{ }$ & $\sqrt{ }$ & $\sqrt{ }$ & $\sqrt{ }$ & & & & & \\
\hline B & $\sqrt{ }$ & $\sqrt{ }$ & & $\sqrt{ }$ & & $\sqrt{ }$ & $\sqrt{ }$ & $\sqrt{ }$ & \\
\hline C & $\sqrt{ }$ & $\sqrt{ }$ & $\sqrt{ }$ & & $\sqrt{ }$ & $\sqrt{ }$ & $\sqrt{ }$ & & $\sqrt{ }$ \\
\hline
\end{tabular}

The example factory is shown in Figure 2. It contains all of the four fundamental types of factory floor agents; queues (Q0, Q3, Q5, Q8, and Q10), material handlers (MH1 and MH7), machines (M2, M4, and M9), and an inspection agent (I6). Notice the MH7 has the option of routing parts along a reentrant pathway. The capabilities for each machine are described below. 


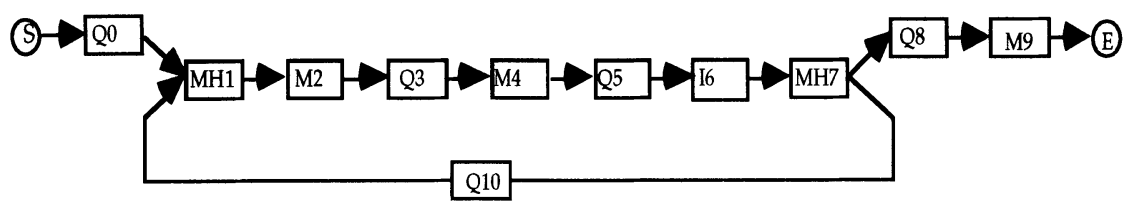

Figure 2 Block diagram of the example factory.

Although varying in length, all the queue agents in this example have similar characteristics. For instance, each queue takes .2 time units to advance a part one position in the queue and 1.3 time units to transfer a part out of the queue. Table 2 shows the specifications for the queues in the example.

Table 2 Queue descriptions.

\begin{tabular}{|l|l|}
\hline Position to position transfer rate & .2 \\
\hline Exit transition time & 1.3 \\
\hline \hline Length of Q0 & 3 \\
\hline Length of Q3 & 3 \\
\hline Length of Q5 & 3 \\
\hline Length of Q8 & 3 \\
\hline Length of Q10 & 10 \\
\hline
\end{tabular}

Two material handlers are present in the system. MH1 can transition a part from one of two sources to a single destination. Conversely, MH7 can transition a part from a single source to one of two destinations. Material handlers will always introduce a point of non-determinism becuase of the multiple sources or destinations. Both machines take a finite amount of time to grasp a part and perform a transport operation. If the destination is currently full, the material handler will attempt to accomplish the grasp, but will wait for the destination to empty before performing transportation operation. Table 3 shows the specific characteristics of the material handlers in this example.

Table 3 Material Handler descriptions.

\begin{tabular}{|l|l|}
\hline Grasp time for MH1 & 1 \\
\hline Transport time for MH1 & 1.3 \\
\hline Grasp time for MH7 & .3 \\
\hline Transport time for MH7 & .7 \\
\hline
\end{tabular}

In the physical system, inspection stations serve to synchronize the knowledge bases with the real world. If the state predicted by the knowledge base and the state of the real world are sufficiently different, a process error may have occurred. Once the knowledge base is updated, the next scheduling phase will begin with a new initial state and reactively adapt to the changes this introduces. Since contingency schedules are not created, the inspection system will not find errors. The inspection station requires 2 time units to inspect a part in the example.

Three machines are present in the system. Each of these machines have different capabilities. M2 can install cards, but it is primarily used for installing the motherboards and power supplies. M4 is a card installation machine only. M9 installs drives only. Each machine can determine if any of its capabilities match those needed by the current part. Machines may perform several sequential operations on the same part without the expense of fixturing and transferring the part between operations. When a part leaves a machine in this example, there is a one time step delay for grasping and transfering the part (not listed in the Table 4. Table 4 gives the capabilities for each machine and process times for the installation of each option. 
Table 4 Machine capabilities.

\begin{tabular}{l|l|l|l|l|l|l|l|l|l} 
Machine & MB & PS & NI & BW & CR & DC & HD & FD & TD \\
\hline M2 & 3.2 & 3 & 2 & 2 & 2 & 2 & & & \\
\hline M4 & & & 3.7 & 3.7 & 3.7 & 3.7 & & & \\
\hline M9 & & & & & & & 4.2 & 4.2 & 4.2 \\
\hline
\end{tabular}

If a part reached M9 in a simulation and did not have all its cards installed, the part would be passed on to the termination point and the simulation would fail. Given the current configuration, there was no way for the cards to be installed before the part exited the assembly line. However, reentrant pathways allow parts to move against the flow of the assembly line. As in the example below, the pathways can also be used to alter the ordering of the parts in the assembly line. Parts with little remaining work can pass jobs with a substantial amount of remaining work to reduce the number of parts in the system.

\subsection{Simulation}

The trace shown in Table 5 is a complete simulation generated in the system described above. For clarity, this simulation is always marked U0. Time $\mathrm{T}=2.4$ is a point of non-determinism. M2 requests to split the simulation because it has to perform one of the following possible operations: install the power supply, install the drive controller, install the monochrome monitor card, or transfer the part out with no further operations. The simulation was split into four duplicate simulations. In each of the duplicates, M2 simulated one of the possible operations. As noted in the comments for that line, the power supply was installed in simulation U0. At simulation time $\mathrm{T}=1.9$, another type of non-determinism occured. MH1 had the choice of drawing the next part from Q0 or Q10. For simulation U0, the part was drawn from Q0. In the alternate simulation, $\mathrm{MH} 1$ will wait for a part to become available from Q10.

At time step 20.6, two event are shown occurring at the same instant. While this is rare, it does not pose a simulation problem because of the decoupled nature of the event processing. Furturemore, notice that workstation $\mathrm{C}$ has chosen to follow the reentrant pathway at $\mathrm{T}=7.9$. Because of the volume of work remaining to be performed on $C$, it would have retarded the progress of workstations A and B. In effect, the smaller jobs passed the larger job.

Table 5 Simulation trace.

\begin{tabular}{|c|c|c|c|c|c|c|c|c|c|c|c|c|c|}
\hline Time & $\mathrm{Q} 0$ & MH1 & M2 & Q3 & M4 & Q5 & 16 & MH7 & Q8 & M9 & Q10 & MIN. & \begin{tabular}{|l|} 
Comment \\
\end{tabular} \\
\hline 0 & 1.9 & $\infty$ & 2.4 & $\infty$ & & $\infty$ & $\infty$ & $\infty$ & $\infty$ & $\infty$ & $\infty$ & \begin{tabular}{|l|}
1.9 \\
\end{tabular} & \\
\hline 1.9 & $\infty$ & 2.9 & & & & & & & & & & 2.4 & $\begin{array}{l}\text { Split } \mathrm{U} 0=0 » 1 \\
\mathrm{Ux}=10 » 1\end{array}$ \\
\hline 2.4 & & & 5.4 & & & & & & & & & 2.9 & $\begin{array}{l}\text { Split } \mathrm{U} 0=\mathrm{PS} \\
\mathrm{Ux}=\mathrm{DC}, \mathrm{BW}, \mathrm{xfer}\end{array}$ \\
\hline 2.9 & & $\infty$ & & & & & & & & & & 3.0 & \\
\hline 3.0 & & & & & 4.0 & & & & & & & 4.0 & $\begin{array}{l}\text { Split U0 }=\text { xfer } \\
\text { Ux= NI,DC }\end{array}$ \\
\hline 4.0 & & & & & $\infty$ & 5.9 & & & & & & 5.4 & \\
\hline 5.4 & & & 6.4 & & & & & & & & & 5.9 & $\begin{array}{l}\text { Split U0=xfer } \\
\mathrm{Ux}=\mathrm{BW}, \mathrm{DC}\end{array}$ \\
\hline 5.9 & & & & & & $\infty$ & 7.9 & & & & & 6.4 & \\
\hline 6.4 & & 7.7 & $\infty$ & 8.3 & & & & & & & & 7.7 & \\
\hline 7.7 & & $\infty$ & 10.9 & & & & & & & & & 7.9 & $\begin{array}{l}\text { Split U0= MB } \\
\text { Ux= xfer }\end{array}$ \\
\hline 7.9 & & & & & & & $\infty$ & 8.9 & & & & 8.3 & $\begin{array}{l}\text { Split } \mathrm{U} 0=7 » 10 \\
\mathrm{Ux}=7 » 8\end{array}$ \\
\hline 8.3 & & & & $\infty$ & 12.0 & & & & & & & 8.9 & $\begin{array}{l}\text { Split U0= BW } \\
\text { Ux }=D C, x \text { fer }\end{array}$ \\
\hline 8.9 & & & & & & & & $\infty$ & & & 12.2 & \begin{tabular}{|l|}
10.9 \\
\end{tabular} & \\
\hline
\end{tabular}




\begin{tabular}{|c|c|c|c|c|c|c|c|c|c|c|c|c|c|}
\hline Time & Q0 & MH1 & $\mathrm{M} 2$ & Q3 & M4 & Q5 & I6 & MH7 & Q8 & M9 & Wh & MIN. & Comment \\
\hline 10.9 & & & 13.9 & & & & & & & & & 12.0 & $\begin{array}{l}\text { Split U0 }=\text { PS } \\
\text { Ux= BW,NI,xfer }\end{array}$ \\
\hline 12.0 & & & & & 15.7 & & & & & & & 12.2 & $\begin{array}{l}\text { Split U0= DC } \\
\text { Ux= xfer }\end{array}$ \\
\hline 12.2 & & 13.2 & & & & & & & & & $\infty$ & 13.2 & $\begin{array}{l}\text { Split U0=10»1 } \\
\text { Ux }=0 » 1\end{array}$ \\
\hline 13.2 & & $\infty t$ & & & & & & & & & & 13.9 & \\
\hline 13.9 & & & 14.9 & & & & & & & & & 14.9 & $\begin{array}{l}\text { Split U0=xfer } \\
\mathrm{Ux}=\mathrm{BW}, \mathrm{NI}\end{array}$ \\
\hline 14.9 & & 16.2 & $\infty$ & 16.8 & & & & & & & & 15.7 & \\
\hline 15.7 & & & & & 16.7 & & & & & & & 16.2 & \\
\hline 16.2 & & $\infty$ & 18.2 & & & & & & & & & 16.7 & $\begin{array}{l}\text { Split U0= NI } \\
\text { Ux= DC, xfer }\end{array}$ \\
\hline 16.7 & & & & & $\infty$ & 18.6 & & & & & & 16.8 & \\
\hline 16.8 & & & & $\infty$ & 20.6 & & & & & & & 18.2 & $\begin{array}{l}\text { Split U0= BW } \\
\text { Ux= NI,xfer }\end{array}$ \\
\hline 18.2 & & & 20.2 & & & & & & & & & 18.6 & $\begin{array}{l}\text { Split U0= DC } \\
\text { Ux }=\text { xfer }\end{array}$ \\
\hline 18.6 & & & & & & $\infty$ & 20.6 & & & & & 20.2 & \\
\hline 20.2 & & & 21.2 & & & & & & & & & 20.6 & \\
\hline 20.6 & & & & & 24.3 & & & & & & & 20.6 & $\begin{array}{l}\text { Split U0= NI } \\
\text { Ux= xfer }\end{array}$ \\
\hline 20.6 & & & & & & & $\infty$ & 21.6 & & & & 21.2 & 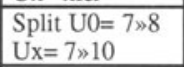 \\
\hline 21.2 & & & $\infty$ & 23.1 & & & & & & & & 21.6 & \\
\hline 21.6 & & & & & & & & $\infty$ & 23.5 & & & 23.1 & \\
\hline 23.1 & & & & $\infty t$ & & & & & & & & 23.5 & \\
\hline 23.5 & & & & & & & & & $\infty$ & 27.7 & & 24.3 & $\begin{array}{l}\text { Split U0 }=\mathrm{HD} \\
\mathrm{Ux}=\mathrm{FD}, \mathrm{xfer}\end{array}$ \\
\hline 24.3 & & & & & 25.3 & & & & & & & 25.3 & \\
\hline 25.3 & & & & 26.6 & $\infty$ & 27.2 & & & & & & 26.6 & \\
\hline 26.6 & & & & $\infty$ & 27.6 & & & & & & & 27.2 & \\
\hline 27.2 & & & & & & $\infty$ & 29.2 & & & & & 27.6 & \\
\hline 27.6 & & & & & $\infty$ & 29.5 & & & & & & 27.7 & \\
\hline 27.7 & & & & & & & & & & 31.9 & & 29.2 & \begin{tabular}{rl|} 
Split U0 & $=$ FD \\
Ux & $=$ xfer \\
\end{tabular} \\
\hline 29.2 & & & & & & & $\infty$ & 30.2 & & & & 29.5 & $\begin{array}{l}\text { Split U0 }=7 » 8 \\
\mathrm{Ux}=7 » 10\end{array}$ \\
\hline 29.5 & & & & & & $\infty$ & 31.5 & & & & & 30.2 & \\
\hline 30.2 & & & & & & & & $\infty$ & 32.1 & & & 31.5 & \\
\hline 31.5 & & & & & & & $\infty$ & 32.5 & & & & 31.9 & 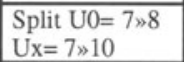 \\
\hline 31.9 & & & & & & & & & & 32.9 & & 32.1 & \\
\hline 32.1 & & & & & & & & & $\infty \dagger$ & & & 32.5 & \\
\hline 32.5 & & & & & & & & $\infty$ & $\infty+t$ & & & 32.9 & \\
\hline 32.9 & & & & & & & & & 34.2 & $\infty$ & & 34.2 & B OUT \\
\hline 34.2 & & & & & & & & & $\infty t$ & 35.2 & & & \\
\hline 35.2 & & & & & & & & & 36.5 & $\infty$ & & 36.5 & A OUT \\
\hline 36.5 & & & & & & & & & $\infty$ & 40.7 & & 40.7 & $\begin{array}{l}\text { Split U0 }=\mathrm{HD} \\
\mathrm{Ux}=\mathrm{TD}, \mathrm{xfer}\end{array}$ \\
\hline 40.7 & & & & & & & & & & 44.9 & & 44.9 & $\begin{array}{l}\text { Split U0= TD } \\
\text { Ux }=\text { xfer }\end{array}$ \\
\hline 44.9 & & & & & & & & 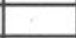 & & 45.9 & & 45.9 & \\
\hline 45.9 & & & & & & & & & & $\infty$ & & $\infty$ & C OUT \\
\hline
\end{tabular}




\section{FUTURE DIRECTIONS AND CONCLUSIONS}

In this work, a new algorithm for distributed simulation and on-line evaluation of schedules has been presented. The algorithm can be applied for multiple machines and is flexible enough to handle most manufacturing scheduling applications. The algorithm is to evaluate heuristics to reduce the complexity of the search procedure. This work has proposed a distributed simulation approach that offers the following advantages: (i) Simulation helps in reducing the number of schedules evaluated to provide a satisfactory response time, (ii) The design of the simulation environment is flexible and can be tailored to the constraints of a factory environment, (iii) The implementation of the distributed simulation environment reflects the requirements foreseen in future schedulers (e.g. scheduling with machines that reason in a variety of ways), and, (iv) Without additional problem-specific heuristics, the system is designed to take a plan and perform a bounded search on generated schedules.

Future research will be directed to additional heuristics, such as: (i) Not allowing parts to cycle through the reentrant pathways unless there is a state change, (ii) Methods for identifying and associating subgoals with parts. These subgoals will determine if a part can transition beyond a specific machine. For instance, in all cards should be installed before a part can enter Q8. (iii) Methods for establishing the initial bound on the simulations. (iv) Alteration of the branching policy to favor attractive solutions.

\section{REFERENCES}

[ChAl86] H. Chochon and R. Alami (1986) "NNS, A Knowledge-Based On-line System For An Assembly Workcell", Proc.of IEEE International Conference on Robotics and Automation, pages 603-9.

[FoKe85] B.R. Fox and K.G. Kempf (1985) "Opportunistic Scheduling for Robotic Assembly", Proc. of IEEE International Conference on Robotics and Automation, pages 880-9.

[Haye93] B. Hayes-Roth (1993) "Opportunistic Control of Action in Intelligent Agents", Proc. of IEEE Transactions on Systems, Man, and Cybernetics, Vol. 23, No. 6, pages 1575-87, November/December.

[Jern95] S.R. Jernigan (1995) "A Reactive, Distributed Simulation Method for Scheduling flow through a Factory Floor", submitted as master's thesis, The University of Texas at Austin, Summer.

[Lyon90] D.M. Lyons (1990) "A Process-Based Approach to Task Plan Representation", Proc. of IEEE International Conference on Robotics and Automation, pages 2142-7.

[MeSa91] L. S. Homem de Mello and A. C. Sanderson (1991) "A Correct and Complete Algorithm for the Generation of Mechanical Assembly Sequences", Proc. of IEEE International Conference on Robotics and Automation, pages 228-40.

[Misr86] J. Misra (1988) "Distributed Discrete-Event Simulation", Computing Surveys, Vol. 18, No. 1, March.

[XiBe88] X. Xia and G.A. Bekey (1988) "SROMA: An Adaptive Scheduler for Robotic Assembly Systems", Proc. of IEEE International Conference on Robotics and Automation, pages 1282-7. 